\title{
Assessing suicidal risk with antiepileptic drugs
}

This article was published in the following Dove Press journal:

Neuropsychiatric Disease and Treatment

13 September 2010

Number of times this article has been viewed

Marco Mula²

Gail S Bell'

Josemir W Sander ${ }^{1,3}$

'Department of Clinical and Experimental Epilepsy, UCL Institute of Neurology, and National Hospital for Neurology and Neurosurgery,

UCL Hospitals NHS Foundation Trust, London, United Kingdom; ${ }^{2}$ Department of Clinical and Experimental Medicine, Division of Neurology, Amedeo Avogadro University, University Hospital Maggiore della Carità, Novara, Italy; ${ }^{3}$ SEIN - Epilepsy Institute in the Netherlands Foundation,

Heemstede, The Netherlands
Correspondence: Ley Sander Department of Clinical and Experimental Epilepsy, UCL Institute of Neurology, PO Box 29, 33 Queen Square, London WCIN 3BG, United Kingdom $\mathrm{Tel}+442031080122$

$\mathrm{Fax}+442031080115$

Email Isander@ion.ucl.ac.uk
Abstract: Recently, the US Food and Drug Administration issued an alert about an increased risk for suicidality during treatment with antiepileptic drugs (AEDs) for different indications, including epilepsy. We discuss the issue of suicide in epilepsy with special attention to AEDs and the assessment of suicide in people with epilepsy. It has been suggested that early medical treatment with AEDs might potentially reduce suicide risk of people with epilepsy, but it is of great importance that the choice of drug is tailored to the mental state of the patient. The issue of suicidality in epilepsy is likely to represent an example of how the underdiagnosis of psychiatric symptoms, the lack of input from professionals (eg, psychologists, social workers, and psychiatrists), and the delay in an optimized AED therapy may worsen the prognosis of the condition with the occurrence of severe complications such as suicide.

Keywords: epilepsy, suicide, adverse effect, depression

\section{The background: suicide in people with epilepsy}

It was first suggested many years ago that, despite popular opinion, suicide occurred "rather frequently" in people with epilepsy. ${ }^{1}$ Since that time increasing evidence has accumulated, ${ }^{2}$ and it seems that the overall risk of committing suicide in people with epilepsy is about 3 times that of the general population. ${ }^{3}$

The relationship between epilepsy and suicide has been investigated in a few studies using a case-control design, mostly in Scandinavia. One of these, using national registries, included every person ascertained as having committed suicide in Denmark during a 17-year period and also up to 20 age-matched and sex-matched controls, still alive, for each person who committed suicide. ${ }^{4}$ Rate ratios, corrected for marital status, labor market status, income, place of residence, and sickness absence were estimated. Of more than 20,000 people who committed suicide, $492(2.3 \%)$ had a history of epilepsy compared with 3,140 (0.7\%) controls (rate ratio, 3.17). Those with epilepsy were 4 times as likely to have a history of psychiatric disease (as listed in the Danish Psychiatric Central Register) as those without epilepsy. In people with no history of psychiatric disease, the adjusted rate ratio of suicide in people with epilepsy was 1.99 (95\% confidence interval [CI], 1.71-2.32) compared with those without epilepsy. The rate ratio of suicide in those with both epilepsy and psychiatric disease was 13.7 compared with those without epilepsy or psychiatric disease. Whether or not psychiatric disease was present in those with epilepsy, the risk of suicide was greatest in the early stages of the condition, usually in the first 6 months after epilepsy diagnosis.

Another case-control study was nested within a cohort study of adults admitted at least once during 1980-1989 period to a hospital in Stockholm County with a diagnosis 
of epilepsy recorded at discharge. ${ }^{5}$ Subsequently, 26 patients committed suicide, and these were matched with 171 patients from the same population who were alive at the end of the study period. A 9-fold increase in risk of suicide associated with psychiatric illness and a similar increase associated with the use of antipsychotic drugs were found. The risk associated with antipsychotic drugs was still increased (almost 4-fold) after adjusting for psychiatric illness and alcohol abuse. In this study, which could look at epilepsy variables, early age of onset of epilepsy was strongly associated with suicide. Both seizure frequency in the previous year and number of AEDs taken showed weak (nonsignificant) relationships with suicide, but numbers of suicides were small.

All suicides that occurred in a 15 -year period in a region of Finland were investigated. ${ }^{6}$ Of 1,877 people who committed suicide, 25 had hospital-treated epilepsy. Those with epilepsy were more likely to have had psychiatric disorders than those without epilepsy.

The cause of the increased risk of suicide associated with epilepsy is not entirely clear. In the past, it was linked to socioeconomic deprivation associated with epilepsy. ${ }^{7}$ More recently, other risk factors have been suggested. Previous suicide ideation or attempt is a risk factor for suicide, ${ }^{2,8}$ so it is also important to assess risk factors for suicidality.

Psychiatric disorders may occur twice as frequently in people with epilepsy as in the general population ${ }^{9}$ and, in the general population, approximately $90 \%$ of people who commit suicide have psychiatric disorders at the time ${ }^{10}$ There is increasing evidence that the comorbidity of depression and epilepsy may be bidirectional, ie, that people with epilepsy may be more prone to developing depression and that people with depression may be more likely to develop epilepsy. ${ }^{11}$ Similarly, a previous attempt to suicide is associated with an increased risk of seizure onset, even after adjusting for depression. ${ }^{11}$ One study in Nigeria compared people with epilepsy, population controls, and a small control group with major depression. A questionnaire was used to identify depression and suicidal ideation. More people with epilepsy had depression than population controls. Suicidal ideation levels were similar in depressed people with epilepsy and in population controls with depression, but suicidal ideation was significantly higher in the controls with major depression. ${ }^{12}$ A questionnaire study from Italy included 103 people with temporal lobe epilepsy, excluding people with current dementia or psychosis. One quarter of people with epilepsy had high levels of hopelessness, suggesting they were at risk of suicide. ${ }^{13}$
A recent study from Brazil attempted to contact all people aged 13 years and older, who had been identified in a previous population-based study of people with epilepsy. ${ }^{14}$ People were asked to complete a questionnaire, and some also had a detailed psychiatric evaluation. Of those who participated (four-fifths of the original cohort), more than one-third reported suicidal ideation, almost one-fifth had made suicidal plans, and $12 \%$ had attempted suicide at least once. Those reporting suicidal thoughts had more anxiety, more depression, and were more likely to be on current mental health treatment. They were also more likely to be female, to be older, to have active epilepsy, and to have visited the emergency department more frequently. ${ }^{14}$ Another study, based largely on the same population, compared 153 people with epilepsy with 154 neighborhood controls. People with epilepsy were more likely to report depression and anxiety, and had significantly raised odds ratios for suicidal thoughts, suicidal plans, and attempted suicide at some stage in life. ${ }^{15}$

All data presented clearly suggest that suicide is overrepresented in people with epilepsy. A recent meta-analysis estimated the overall SMR for suicide in people with epilepsy as 3.3 (95\% CI, 2.8-3.7). The SMR, however, ranges between 2.1 for people with newly diagnosed epilepsy in the community and 13.9 in those following temporal lobe resections. ${ }^{3}$ This is probably due to a number of reasons that are both biological and psychosocial. More data are warranted, and well-designed studies are needed to identify and isolate relevant variables.

\section{The assessment of suicide risk in clinical practice}

In psychiatric practice, it is generally agreed that mental disorders increase the risk for suicide, especially when substance abuse or depressive symptoms coexist with another psychiatric disorder or condition. ${ }^{16} \mathrm{~A}$ number of psychosocial factors are also associated with risk for suicide and suicide attempts. ${ }^{17}$ These include recent life events such as losses, especially employment, career, finance, housing, marital relationship, physical health, and a sense of a future, and chronic or long-term problems such as relationship difficulties, unemployment, and problems with the legal authorities. Psychological states of acute distress (especially humiliation, despair, guilt and shame) are often present in association with suicidal ideation, planning, and attempts. Although not uniformly predictive of suicidal ideation and behavior, they are warning signs of psychological vulnerability and indicate 
a need for mental health evaluation to minimize immediate discomfort and to evaluate suicide risk. Nevertheless, these risk factors are not necessarily closely related in time to the onset of suicidal behaviors - nor does any risk factor alone increase or decrease risk. Population-based research suggests that the risk for suicide increases with an increase in the number of risk factors present such that when more risk factors are present at any one time, the more likely it is that they indicate an increased risk for suicidal behaviors at that time. ${ }^{18}$

Are there warning signs for clinicians about suicide? There are 3 major pointers as follows: threatening to hurt or kill self; looking for ways to kill self such as seeking access to drugs, weapons, or other means; talking or writing about death, dying, or suicide. ${ }^{19}$ The remaining list of warning signs should alert the clinician that a mental health evaluation needs to be conducted in the very near future and that precautions need to be put into place immediately to ensure the safety, stability, and security of the individual: hopelessness; rage, anger, seeking revenge; acting recklessly or engaging in risky activities, seemingly without thinking; feeling trapped, like there is no way out; increasing alcohol or drug abuse; withdrawing from friends, family, or society; anxiety, agitation, being unable to sleep or sleeping all the time; dramatic changes in mood; feeling no reason for living; and having no sense of purpose in life. Other behaviors that may be associated with increased shortterm risk for suicide include the patient making arrangements to divest responsibility for dependent others (children, pets, elders) or making other preparations such as updating wills, making financial arrangements for paying bills, saying farewell to loved ones. ${ }^{17,19}$

It is important to emphasize at this point that a number of specific variables may increase or decrease the risk of suicide. ${ }^{17,19}$ They do not necessarily impart a causal relationship but rather they serve as guidelines for the clinician to weigh the relative risk of an individual engaging in suicidal behaviors within the context of the current clinical presentation and psychosocial setting. In fact, individuals differ in the degree to which risk and protective factors affect their propensity for engaging in suicidal behaviors, and, within an individual, the contribution of each risk and protective factor to their suicidality will vary over the course of their lives. No one risk factor, or set of risk factors, necessarily conveys increased suicidal risk. Nor does one protective factor, or set of protective factors, insure protection against engagement in suicidal behaviors. Furthermore, because of their different statistical correlations with suicidal behaviors, these factors are not equal and one cannot "balance" one set of factors against another in order to derive a total score of relative suicidal risk. Some risk factors are unchallengeable (eg, age, gender, race/ethnicity), whereas others are more situation-specific (eg, loss of housing, exacerbation of pain in a chronic condition, and onset or exacerbation of psychiatric symptoms).

Ideally, with the elucidation and knowledge of an individual's risk and protective factors as a backdrop, the sensitive clinician will inquire about the individual's reasons for living and for dying to better evaluate current risk for suicide.

Factors that may increase a person's risk have been mentioned already. Conversely, those that may decrease the risk for suicide are called protective factors and include the following: positive social support; spirituality; sense of responsibility to family; children in the home, pregnancy; life satisfaction; reality testing ability; positive coping skills; positive problem-solving skills; positive therapeutic relationship.

\section{The US Food and Drug Administration meta-analysis}

In 2005, the US Food and Drug Administration (FDA) became concerned that there may be an excess risk of suicidal ideation or behavior associated with the use of AEDs. They requested that sponsors of AED studies submit data from placebo-controlled trials and then performed a metaanalysis of suicidal behavior and ideation based on previously defined criteria. Results were published in 2008. ${ }^{20}$ Eleven AEDs, used for any indication, were involved in the 199 placebo-controlled trials, with more than 27,000 patients taking AEDs and 16,000 taking placebo. Most of the trials were of short duration (mean 89 days; maximum 582 days) and one-fourth of patients were in epilepsy trials, just over one-fourth in trials with psychiatric indications and almost $50 \%$ in trials for "other" indications (including agitation, pain, obesity, and tremor). Two newer AEDs (topiramate $27 \%$ and pregabalin $24 \%$ ) accounted for $50 \%$ of the patients in the trials, and older AEDs for very few (carbamazepine $1 \%$, valproate $5 \%$, and phenytoin and phenobarbital were not included in any of the trials).

Four people committed suicide (all on AEDs and none on placebo). In the AED group, $0.37 \%$ of patients had suicidal behavior or ideation compared with $0.24 \%$ in the placebo group; most events, however, were suicidal ideation. The overall odds ratio for suicidal behavior or ideation was 1.8 (95\% CI, 1.24-2.66). The odds ratios were significantly increased for topiramate and lamotrigine and were (nonsignificantly) decreased for carbamazepine and valproate. The odds ratios were significantly increased for 
suicidal behavior or ideation in trials for epilepsy indications but not in trials for psychiatric or other indications. It should be noted, however, that in most epilepsy trials, the AED was not used in monotherapy, whereas in trials with psychiatric or other indications, the AED was used in monotherapy in $75 \%-86 \%$ of trials.

As a result of the meta-analysis, the FDA issued an alert about the use of AEDs, in which they state that any patients taking AEDs should be monitored for any suggestion of suicidal thoughts or behavior, or depression. They also state that patients and their families and caregivers should be warned that AEDs may increase the risk of suicidal thoughts or actions and that manufacturers of AEDs should include a warning in the drug labeling to this effect. ${ }^{21}$

Following the FDA meta-analysis, further studies have attempted to investigate the relationship between AEDs and suicide. A study from Denmark using national registers of prescriptions, comorbidity, psychiatric diagnoses, and cause of death used 2 different methodologies to investigate this relationship. ${ }^{22}$ In the first analysis, each individual who committed suicide during a 10 -year period and who had also taken AEDs during that 10-year period was considered. The control for each person who committed suicide was that same individual during a control period of 30 days, 2 (or 3) months before the suicide. Thus, the number of people taking AEDs during the 30-day period prior to the suicide was compared with the number of the same people who took AEDs in a 30-day period 2 (or 3) months earlier. Overall, AED usage increased the risk of suicide, as did the use of clonazepam, valproate, lamotrigine, or phenobarbital. The second analysis included only people who started taking AEDs during the 10 -year period and who had not been prescribed AEDs in the year prior to the 10-year period. Survival analysis of suicide during the first 6 months of exposure to an AED found hazard ratios that were increased (compared with carbamazepine) for clonazepam, valproate, lamotrigine, phenobarbital, and levetiracetam. It is pointed out that only a minority of the cohort was registered as having epilepsy and that individuals may have restarted AED treatment in response to worsening of underlying psychiatric or painrelated symptoms. It is interesting that valproate appeared to increase the risk of suicide, which was not the case in the FDA meta-analysis.

Investigators in the United States used Veterans Health Administration data to assess suicide-related behaviors in people with a new prescription for monotherapy AED. For each person aged 66 years and older with new AED treatment, who had suicide-related behavior, 12 controls were found, matched according to a previous history of suicide-related behavior, the year of first AED prescription, and a diagnosis of epilepsy. Gabapentin was used as the reference AED, as more than $75 \%$ of individuals received this drug. Compared with gabapentin, there was a slight increase in suicide-related behaviors in those taking the newer AEDs (levetiracetam or lamotrigine), but the confidence intervals were wide. The strongest predictor for suicide-related behavior was found to be having an affective disorder diagnosed prior to the AED prescription. In this group with new AED prescriptions, the FDA's finding that suicidal behavior was more common in those with epilepsy than in those using AEDs for other indications was not confirmed. ${ }^{23}$

Another US cohort study using the HealthCore Integrated Research Database reported an increased risk of suicidal attempts and completed suicide or violent death in patients taking gabapentin, lamotrigine, oxcarbazepine, tiagabine and valproate compared with topiramate ${ }^{24}$ regardless of a specific indication. Only a small proportion of the cohort was taking AEDs for epilepsy, ranging from $0.3 \%$ to $19 \%$, and the median follow-up was 60 days. In the epilepsy subanalysis (compared with carbamazepine), it seemed that gabapentin was associated with an increased suicidal risk. In common with other studies in the field, the lack of pretreatment data represents a major limitation.

\section{Do AEDs increase or reduce the risk of suicide in epilepsy?}

It has been suggested that the concern of the FDA over all AEDs might be excessive, particularly as the meta-analysis only found statistically significant effects for 2 AEDs. ${ }^{25,26}$ The methodology of using only spontaneously reported suicidality events has been questioned. ${ }^{26}$ It has also been suggested that the risk of uncontrolled seizures (due to stopping or not starting AEDs) might well be in excess of the risk of suicidality. ${ }^{26,27}$ The rate of suicide is almost certainly increased in people with epilepsy, ${ }^{3,27}$ but the number of deaths due to suicide in people with epilepsy is much smaller than those due to accidents or Sudden Unexpected Death in Epilepsy which seem to be related to the frequency of seizures. ${ }^{26}$

A study using the UK General Practice Research Database investigated people with incident epilepsy (defined as a diagnostic code of epilepsy and at least 2 AED prescriptions) and up to 4 matched controls for each person with incident epilepsy. With more than 3,000 people with epilepsy and 11,000 controls, the incidence rate ratio (IRR) was significantly increased for suicide attempt before the diagnosis of epilepsy and for the first year after the diagnosis. ${ }^{28}$ Looking at suicide recurrence in subjects with a previous history of suicide attempt, however, the IRR was actually decreased after epilepsy diagnosis and epilepsy drug 
treatment was started, suggesting that AEDs actually decrease the risk of suicide.

Data from the RANSOM study suggested that nonadherence to AEDs can have serious or fatal consequences for patients with epilepsy with a 3-fold increase in mortality risk compared with patients who are adherent to drug treatment. ${ }^{29}$

Emerging data from the field of psychiatry are of particular interest. A US pharmacoepidemiologic study using the PharMetrics medical claims database reported that AEDs do not increase the risk of suicide attempts in patients with bipolar disorder compared with those not treated with AEDs or lithium. ${ }^{30}$ Conversely, the use of AEDs reduced suicide attempt rates both relative to patients not receiving any psychotropic medication and relative to their pretreatment levels.

All these findings taken together clearly suggest that advantages from an appropriate treatment of seizures in people with epilepsy clearly overcome potential disadvantages, making AEDs potentially protective agents against suicide. In agreement with others, we suggest that early medical treatment with AEDs could potentially reduce the suicide risk of people with epilepsy because of the mood-stabilizing properties of some compounds, ${ }^{4}$ but it becomes even more important that the drug choice needs to be tailored to the mental state of the patient. The choice of a specific AED is influenced by a number of variables including, but not restricted to, the type of seizure or the epilepsy syndrome. Side-effect profile of the drug, individual circumstances of the patient including the mental state, affordability, and personal preferences need to be taken into account.

The prognosis of epilepsy and the occurrence of severe complications, including suicide, may be made worse by missing important comorbidity and by delaying or withholding treatment. These issues may be compounded by the lack of input from professionals such as psychologists, social workers, and psychiatrists.

\section{Disclosures}

This work was supported by UCLH/UCL who received a proportion of funding from the Department of Health's NIHR Biomedical Research Centers funding scheme.

Drs Sander and Mula have received travel grants or consultancy fees from various pharmaceutical companies, including Novartis, Pfizer, UCB Pharma, Eisai, Schwarz Pharma, Janssen-Cilag, sanofi-aventis, and GSK, involved in the manufacture of antiepileptic drugs. Dr Bell's husband works for, and has shares in, GSK, which manufactures lamotrigine.

\section{References}

1. Prudhomme C. Epilepsy and suicide. J Nerv Ment Dis. 1941;94:722-727.

2. Harris EC, Barraclough B. Suicide as an outcome for mental disorders. A meta-analysis. Br J Psychiatry. 1997;170:205-228.

3. Bell GS, Gaitatzis A, Bell CL, Johnson AL, Sander JW. Suicide in people with epilepsy: how great is the risk? Epilepsia. 2009;50(8):1933-1942.

4. Christensen J, Vestergaard M, Mortensen PB, Sidenius P, Agerbo E. Epilepsy and risk of suicide: a population-based case-control study. Lancet Neurol. 2007;6(8):693-698.

5. Nilsson L, Ahlbom A, Farahmand BY, Asberg M, Tomson T. Risk factors for suicide in epilepsy: a case control study. Epilepsia. 2002;43(6): 644-651.

6. Mainio A, Alamaki K, Karvonen K, Hakko H, Sarkioja T, Rasanen P. Depression and suicide in epileptic victims: a population-based study of suicide victims during the years 1988-2002 in northern Finland. Epilepsy Behav. 2007;11(3):389-393.

7. Suicide and epilepsy. Br Med J. 1980;281(6239):530.

8. Jones JE, Hermann BP, Barry JJ, Gilliam FG, Kanner AM, Meador KJ. Rates and risk factors for suicide, suicidal ideation, and suicide attempts in chronic epilepsy. Epilepsy Behav. 2003;4(Suppl 3):S31-S38.

9. Gaitatzis A, Carroll K, Majeed A, Sander JW. The epidemiology of the comorbidity of epilepsy in the general population. Epilepsia. 2004;45(12):1613-1622.

10. Barraclough B, Bunch J, Nelson B, Sainsbury P. A hundred cases of suicide: clinical aspects. Br J Psychiatry. 1974;125(0):355-373.

11. Hesdorffer DC, Hauser WA, Olafsson E, Ludvigsson P, Kjartansson O. Depression and suicide attempt as risk factors for incident unprovoked seizures. Ann Neurol. 2006;59(1):35-41.

12. Okubadejo NU, Danesi MA, Aina OF, Ojini FI, Adeyemi JD, Olorunshola DA. Prospective case-control study of interictal depression and suicidal ideation in Nigerians with epilepsy. Niger Postgrad Med J. 2007;14(3):204-208.

13. Pompili M, Vanacore N, Macone S et al. Depression, hopelessness and suicide risk among patients suffering from epilepsy. Ann Ist Super Sanita. 2007;43(4):425-429.

14. Stefanello S, Marin-Leon L, Fernandes PT, Min LL, Botega NJ. Suicidal thoughts in epilepsy: a community-based study in Brazil. Epilepsy Behav. 2010;17(4):483-488.

15. Stefanello S, Marin-Leon L, Fernandes PT, Li LM, Botega NJ. Psychiatric comorbidity and suicidal behavior in epilepsy: a community-based case-control study. Epilepsia. 2010;51(7): $1120-1125$

16. Maris RW, Berman AL, Silverman MM. Comprehensive Textbook of Suicidology. New York: The Guilford Press; 2000.

17. Jacobs DG. The Harvard Medical School Guide to Suicide Assessment and Intervention. San Francisco, CA: Jossey-Bass; 1999.

18. Kessler RC, Borges G, Walters EE. Prevalence of and risk factors for lifetime suicide attempts in the National Comorbidity Survey. Arch Gen Psychiatry. 1999;56(7):617-626.

19. Rudd MD, Berman AL, Joiner TE, et al. Warning signs for suicide: theory, research and clinical applications. Suicide Life Threat Behav. 2006;36(3):255-262.

20. US Food and Drug Administration. Antiepileptic drugs and suicidality. Available from: http://www.fda.gov/ohrms/dockets/ac/08/briefing/20084372b1-01-FDA.pdf. Accessed May 23, 2008.

21. US Food and Drug Administration. Information for Healthcare Professionals: Suicidal Behavior and Ideation and Antiepileptic Drugs [updated 2010 March 3]. Available from: http://www.fda.gov/Drugs/ DrugSafety/PostmarketDrugSafetyInformationforPatientsandProvide rs/ucm100192.htm. Accessed Dec 16, 2008.

22. Olesen JB, Hansen PR, Erdal J, et al. Antiepileptic drugs and risk of suicide: a nationwide study. Pharmacoepidemiol Drug Saf. 2010;19(5): 518-524.

23. VanCott AC, Cramer JA, Copeland LA, et al. Suicide-related behaviors in older patients with new anti-epileptic drug use: data from the VA hospital system. BMC Med. 2010;8:4. 
24. Patorno E, Bohn RL, Wahl PM, et al. Anticonvulsant medications and the risk of suicide, attempted suicide, or violent death. JAMA. 2010;303(14): 1401-1409.

25. Kanner AM. Suicidality and epilepsy: a complex relationship that remains misunderstood and underestimated. Epilepsy Curr. 2009;9(3): 63-66.

26. Hesdorffer DC, Kanner AM. The FDA alert on suicidality and antiepileptic drugs: Fire or false alarm? Epilepsia. 2009;50(5):978-986.

27. Bell GS, Mula M, Sander JW. Suicidality in people taking antiepileptic drugs: What is the evidence? CNS Drugs. 2009;23(4):281-292.
28. Hesdorffer DC, Ishihara-Paul L, Mynepalli L, Webb DJ, Weil J, Hauser WA. Epilepsy and psychiatric disorders: evidence for a bidirectional relationship. Epilepsia. 2009;50(S11):220-221

29. Faught E, Duh MS, Weiner JR, Guérin A, Cunnington MC. Nonadherence to antiepileptic drugs and increased mortality: findings from the RANSOM Study. Neurology. 2008;71(20):1572-1578.

30. Gibbons RD, Hur K, Brown CH, Mann JJ. Relationship between antiepileptic drugs and suicide attempts in patients with bipolar disorder. Arch Gen Psychiatry. 2009;66(12):1354-1360.

\section{Publish your work in this journal}

Neuropsychiatric Disease and Treatment is an international, peerreviewed journal of clinical therapeutics and pharmacology focusing on concise rapid reporting of clinical or pre-clinical studies on a range of neuropsychiatric and neurological disorders. This journal is indexed on PubMed Central, the 'PsycINFO' database and CAS, and is the official journal of The International Neuropsychiatric Association (INA). The manuscript management system is completely online and includes a very quick and fair peer-review system, which is all easy to use. Visit http://www.dovepress.com/testimonials.php to read real quotes from published authors.

Submit your manuscript here: http://www.dovepress.com/neuropsychiatric-disease-and-treatment-journal 